\title{
Moths and butterflies (Lepidoptera) of the continental part of the Nenets Autonomous Okrug, Russia
}

\author{
Mikhail V. Kozlov, Jaakko Kullberg \& Vitali Zverev
}

\begin{abstract}
Kozlov, M. V., Kullberg, J. \& Zverev, V. 2019: Moths and butterflies (Lepidoptera) of the continental part of the Nenets Autonomous Okrug, Russia. - Entomol. Fennica 30: 72-89. https://doi.org/10.33338/ef.82923
\end{abstract}

Until very recently, Nenets Autonomous Okrug (NAO), located in the northeastern part of European Russia, was the least studied region of Russia in terms of its moth fauna. Intensive sampling in the surroundings of Naryan-Mar, combined with critical revision of earlier publications and evaluation of museum collections, resulted in the discovery of a relatively rich fauna of Lepidoptera. The first regional checklist of moths and butterflies of the continental part of NAO includes 324 species (169 species of microlepidoptera and 155 species of macrolepidoptera), 178 of which are reported from NAO for the first time. We estimate that 40 to 180 species remain to be found in the study region. The recorded species mostly belong to residents of northern boreal forests and bogs. The fauna of moths and butterflies of NAO clearly differs from the fauna of Fennoscandia, due to the relatively higher proportion of East Palaearctic and Beringian species.

\begin{abstract}
M. V. Kozlov \& V. Zverev, Department of Biology, University of Turku, FI-20014 Turku,Finland; E-mails:mikoz@utu.fi \& vitzve@utu.fi

J. Kullberg, Harmaahaikarankuja 3 i 9, FI-00940 Helsinki, Finland; E-mail: jaakko.kullberg@gmail.com
\end{abstract}

Received 16 September 2018, accepted 11 October 2018

\section{Introduction}

Historically, insect collecting has been a widespread endeavour, and it became a very popular educational hobby in the Victorian age. However, the rapid development of molecular research techniques has made the entomologist, who hunts insects in the wild to learn their distribution and biology, not only comical but also old-fashioned. Unfortunately, this opinion is shared not only by the general public, but also by decision-makers, who steadily and permanently decrease the funding of natural history museums.

At the same time, the importance of biodiversity for ecosystem functions and, consequently, for mankind, is not questioned. However, there is a risk that a large part of the Earth's biodiversity will become extinct before it is described (Hanski et al. 1995). Similarly, the distribution of organisms is currently undergoing modifications at an increasing rate due to both globalisation and environmental change, and especially climate warming (Parmesan et al. 1999, Hill et al. 2002, Early et al. 2016). Consequently, we are in danger of facing irreversible losses of as yet undocumented distribution patterns of organisms - patterns, which were formed during the millennia before humans started to dominate the Earth. This especially concerns the Polar regions, where climatic change appears disproportionately rapid (Walther et al. 2002, Doney et al. 2012, Zvereva et al. 2016). 


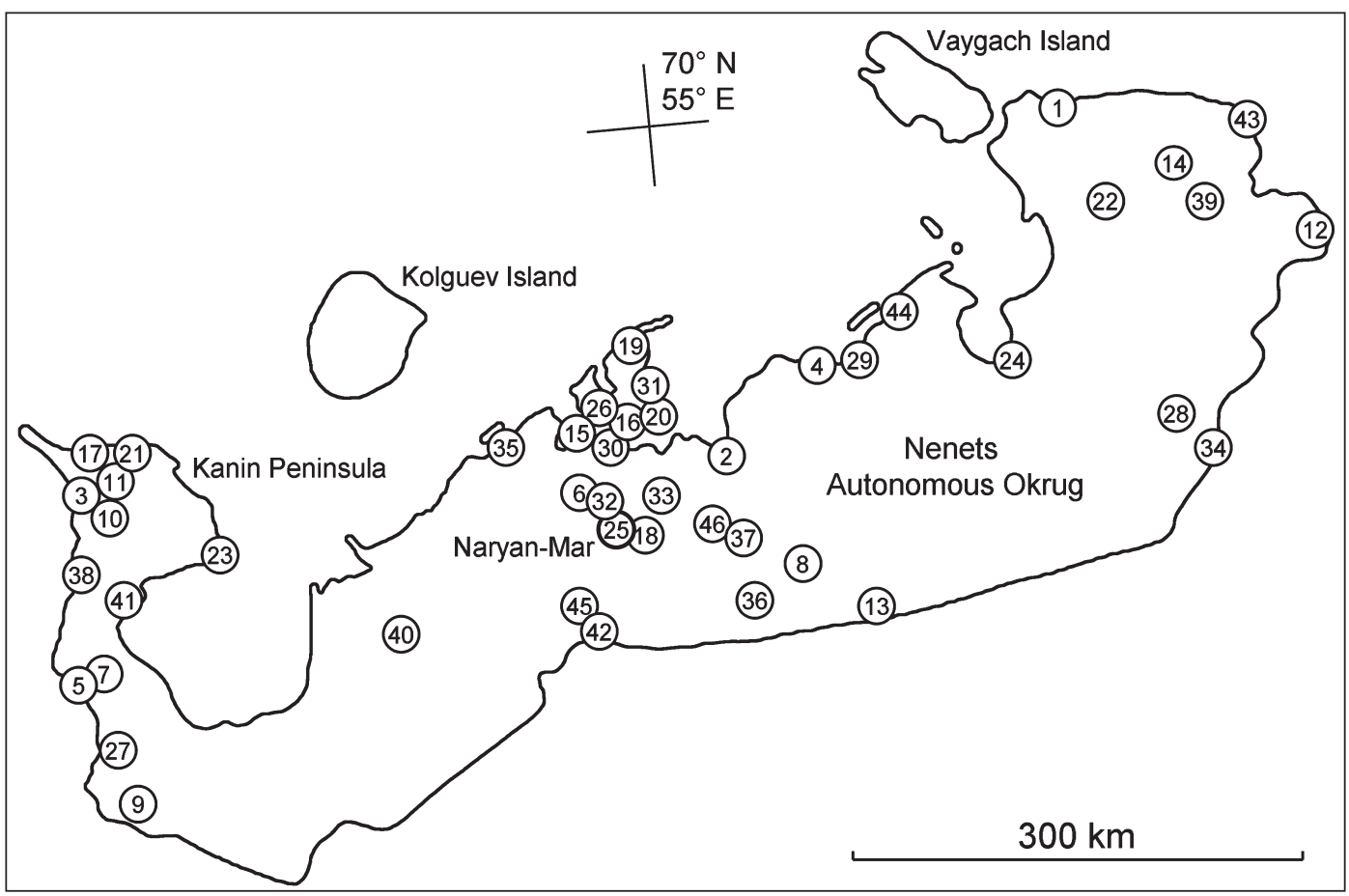

Fig. 1. Sampling localities in the continental part of the Nenets Autonomous Okrug: 1, Amderma; 2, Bolvanskaya Guba; 3, Bugryanitsa; 4, Chernaya; 5, Chizha; 6, Golodnaya Guba; 7, Gorby; 8, Jangech-Mylk; 9, Kabanova; 10, Kambalnitsa; 11, Kaninskij Kamen'; 12, Kara; 13, Kharyaginskij; 14, Khubtape; 15, Kolokolkova; 16, Korovinskaya; 17, Krynka; 18, Kuja; 19, Kuznetskaya; 20, Lovetskij; 21, Madakha; 22, Malaya Padeya; 23, Mikulkin; 24, More-Yu; 25, Naryan-Mar; 26, Nenetskaya Gryada; 27, Nes'; 28, Padimey; 29, Pakhancheskaya Guba; 30 , Pechora site 2; 31, Pechora site 3; 32, Pechora site 4; 33, Pechora site 7; 34, Seida; 35, Sengeiskij; 36, Shapkina, middle flow; 37, Shapkina, upper flow; 38, Shoina; 39, Sibirchatayaha; 40, Svetlaya; 41, Syatorei; 42, Toshviska; 43, Ust-Kara; 44, Varandey; 45, Velikovisochnoye; 46, Vesniyu. For the position of the Nenets Autonmous Okrug in Russia, see Fig. 3b.

Arctic habitats have always fascinated entomologists, although only few of these researchers were ready to spend several months in hostile and mosquito-infested tundra environments to collect a few dozen moths and butterflies (Kullberg et al. 2013). Therefore, the faunistic data on Lepidoptera from the Arctic are generally scarce. Nevertheless, a surprising observation is that the least studied (in terms of moth and butterfly fauna) region of Russia lies in Europe, rather than in remote parts of Siberia: only 108 species have been reported in the Catalogue of Russian Lepidoptera from the Nenets Autonomous Okrug (NAO hereafter) and the Novaya Zemlya archipelago (Sinev 2008), a region of $267,350 \mathrm{~km}^{2}$.

Of course, the diversity of Lepidoptera is extremely low in Polar regions: the fauna of the relatively well-known Svalbard Archipelago in- cludes only three breeding moth species (Coulson 2007), and only four species of Lepidoptera were found on Bolshevik Island in the Severnaya Zemlya Archipelago (Makarova et al. 2013). However, the continental part of NAO lies at about the same latitudes as the Kola Peninsula, which houses at least 813 species of Lepidoptera (Kozlov \& Kullberg 2010). We therefore hypothesised that the low number of species recorded from NAO reflects an insufficient collecting effort rather than a faunistic poverty in this region. We tested this hypothesis by conducting insect collection in the surroundings of Naryan-Mar. We also revised the historical records (in particular, those by Poppius 1906) and accounted for faunistic information on Lepidoptera published during the past decades. Most of this published material has been written in Russian, which has 

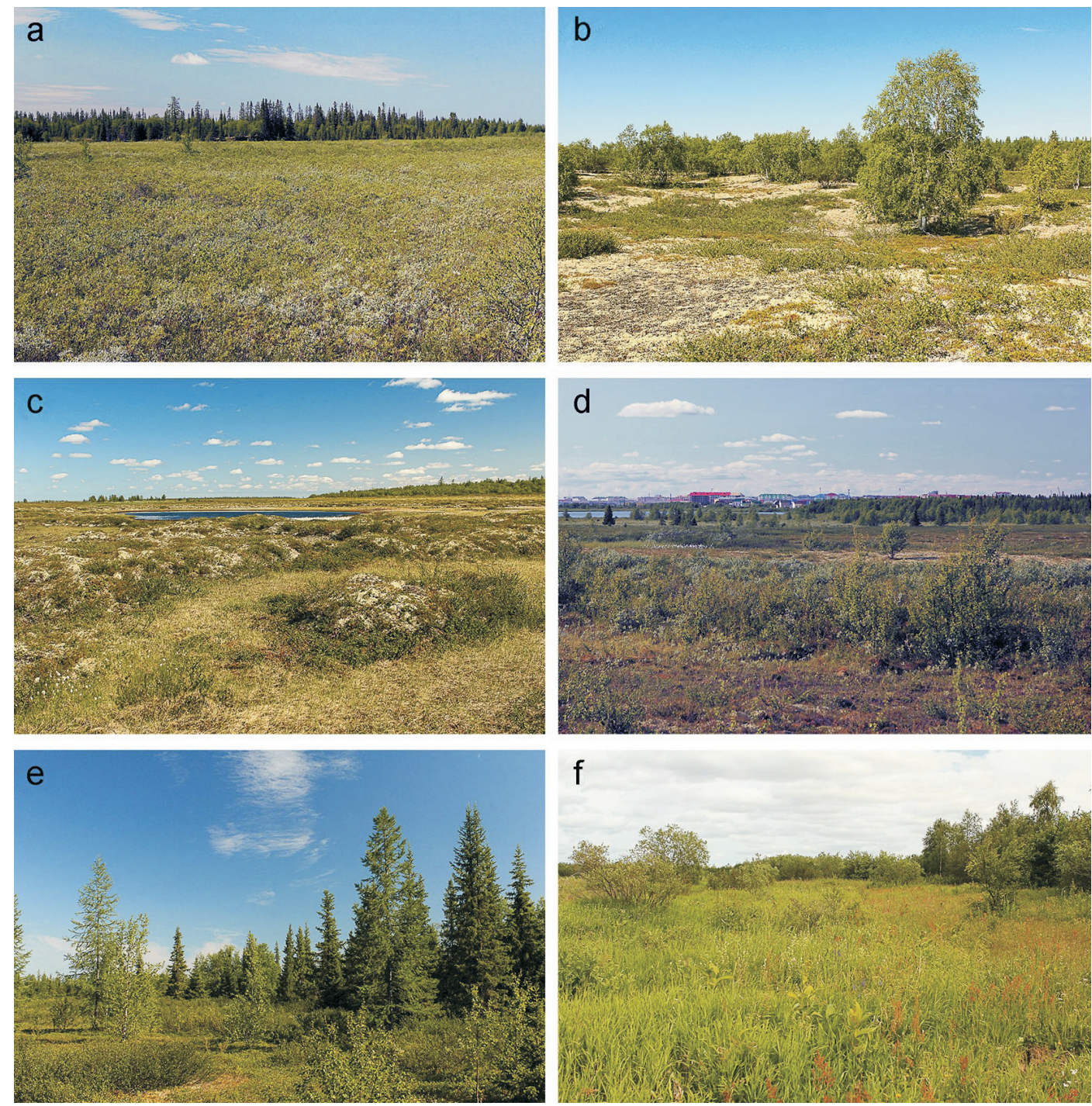

Fig. 2. Habitats in surroundings of Naryan-Mar. - a. Erect dwarf-shrub wet tundra in front of spruce forest. - b. Low-shrub dry tundra at the border of birch woodland. - c. Low-shrub tundra and sedge wetland. $-\mathrm{d}$. Mixture of habitat patches at the outskirts of Naryan-Mar. $-\mathrm{e}$. Mixed forest formed by Norway spruce, larch and birch. $-\mathrm{f}$. Meadow along a riverbank.

restricted its availability to the international scientific community. This published information (Ross 2000, Tatarinov \& Kulakova 2005, 2010, 2013, Bolotov 2012, Vlasova et al. 2014, Tatarinov 2016) concerns butterflies and larger moths primarily, whereas records of so-called "microlepidoptera" has remained scarce (but see Kullberg et al. 2013).

In this paper, we summarise the recent knowledge of the moths and butterflies of the continental part of NAO. A complementary study of Lepi- doptera of the Russian Arctic islands located in the Barents Sea will soon be published (Kullberg et al. 2019). We report our main results in a form of a checklist that includes all species recorded from NAO and all localities from which each species has been collected. We believe that, despite its incompleteness, this list may prove useful for ecological and biographical research and will facilitate further studies of Lepidoptera in Polar regions by elucidating taxonomic and geographic gaps in the present knowledge. 


\section{Materials and methods}

\subsection{Study region}

The continental part of NAO (Fig. 1) covers approximately $170,000 \mathrm{~km}^{2}$ (slightly more than the Baltic States: Lithuania, Latvia and Estonia together), but has a human population of only 42,000. One half of its inhabitants live in the town of Naryan-Mar, the only settlement in NAO which has scheduled flight connections with Moscow and St. Petersburg. NAO is located in three vegetation zones: tundra ( $77 \%$ of the territory), forest-tundra (15\%) and northern taiga forests $(8 \%)$. The average air temperatures in NAO range from -17 to $-19^{\circ} \mathrm{C}$ in December and from 7 to $10^{\circ} \mathrm{C}$ in July. The larger part of NAO belongs to the border security zone, so visiting these restricted areas requires a special permit.

\subsection{Collecting}

Our study is primarily based on the material collected around Naryan-Mar on 2-12 July 2013 by M.V.K. and V.Z. and on 3 July-1 August 2015 by A. Stekolstchikov. Occasional sampling was also performed in Toshviska, Velikovisochnoe and Lovetskij by T. Dyaditsyna (in 2014-2016) and in Bolvanskaja Guba and Pakhancheskaya Guba by O. Makarova (18-28 July 2015). In combination with the published data, this yielded a total of 46 collecting localities (Fig. 1, Table 1).

In the surroundings of Naryan-Mar (67\%38' $\mathrm{N}, 53^{\circ} 00^{\prime} \mathrm{E}$ ), which is located on the bank of the Pechora River, we collected moths and butterflies in the following habitats: (1) erect dwarf-shrub tundra with a dense cover of Betula nana (up to $80 \mathrm{~cm}$ tall) and several species of willows, including Salix lanata (Fig. 2a); (2) low-shrub tundra with a sparse cover of $B$. nana $(20-30 \mathrm{~cm}$ tall), dwarf shrubs (Vaccinium uliginosum, $V$. vitis-idaea, Arctous alpina) and lichens (Cladonia spp.) (Fig. 2b); (3) sedge wetlands, which included swampy areas covered by Sphagnum mosses, sedges (Eriophorum angustifolium, E. vaginatum, Carex paupercula) and Comarum palustre, with small spots of open water, and drier areas covered by Andromeda polifolia, Rubus chamaemorus and B. nana (Fig. 2c, d); (4) downy birch
(B. pubescens) woodland on sandy soils, sometimes with larch (Larix sibirica), with spots of lichens scattered among sparse field layer vegetation consisting of Empetrum hermaphroditum, Arenaria sp., Tanacetum bipinnatum, Allium schoenoprasum var. sibiricum and Campanula sp. (Fig. 2b); (5) forest islands formed by Norway spruce (Picea abies), larch and downy birch, with a dense understory consisting of Juniperus sibirica, Lonicera pallasii, Rosa sp., Ribes rubrum and diverse herbaceous vegetation, including Chamenerion angustifolium, Veratrum lobelianum, Solidago virgaurea, Geranium sylvaticum, Trollius europaea, Rubus arcticus, Thalictrum minus, Aquilegia sp., Trifolium pratense, Tanacetum bipinnatum, Allium schoenoprasum var. sibiricum, Clematis sibirica, V. uliginosum, V. vitis-idaea, Polygonum viviparum, Galium boreale, Equisetum arvense, Valeriana $\mathrm{sp}$. and Linnaea borealis (Fig. 2a, e); and (6) relatively narrow, 10-50 m wide, meadows that extended along riverbanks and were covered primarily by $C$. angustifolium, V. lobelianum, T. vulgaris, $P$. viviparum, Achillea millefolium, Filipendula ulmaria, Veronica sp., Alchemilla sp. and Galium boreale (Fig. 2f).

The insects were collected by netting; the total collecting time by M.V.K. and V.Z. was ca. 140 person-hours and by A. Stekolstchikov ca. 80 person-hours. We attempted light trapping in Naryan-Mar, but did not capture even a single moth during a three-night session. We also recorded easily identifiable species based on visual observations, collected leaf mines and reared moths from field-collected larvae. The pinned specimens are mostly deposited in the Zoological Museum, University of Helsinki, Finland $(\mathrm{MZH})$; about one half of the samples collected in 2013 was donated to the Natural History Museum (London). The plant leaves with insect mines are deposited in the Naturalis Biodiversity Center, Leiden, the Netherlands (RMNH).

\subsection{Revision of earlier records}

The specimens collected by Poppius (1906) in $\mathrm{NAO}$ are deposited in the MZH; among them, we examined all specimens, which, in our opinion, may have been misidentified. We checked the collections of the Zoological Institute in St. Pe- 
Table 1. List of sampling localities in the continental part of the Nenets Autonmous Okrug ${ }^{1}$.

\begin{tabular}{|c|c|c|c|c|}
\hline Locality name and type & $\begin{array}{l}\text { Lati- } \\
\text { tude, N }\end{array}$ & $\begin{array}{l}\text { Longi- } \\
\text { tude, E }\end{array}$ & $\begin{array}{l}\text { No of } \\
\text { species }^{2}\end{array}$ & Data sources \\
\hline Amderma (village) & $69^{\circ} 45^{\prime}$ & $61^{\circ} 40^{\prime}$ & $0 / 35$ & $\begin{array}{l}\text { Kullberg et al. (2013), Vlasova et al. (2014), } \\
\text { Tatarinov (2016) }\end{array}$ \\
\hline Bolvanskaya Guba (bay) & $68^{\circ} 05^{\prime}$ & $54^{\circ} 47^{\prime}$ & $1 / 0$ & O. Makarova leg. (2015) \\
\hline Bugryanitsa (river) & $68^{\circ} 14^{\prime}$ & $44^{\circ} 14^{\prime}$ & $0 / 4$ & Poppius (1906) \\
\hline Chernaya (river) & $68^{\circ} 35^{\prime}$ & $56^{\circ} 35^{\prime}$ & $0 / 16$ & Tatarinov (2016) \\
\hline Chizha (river) & $67^{\circ} 05^{\prime}$ & $44^{\circ} 22^{\prime}$ & $0 / 1$ & Poppius (1906) \\
\hline Golodnaya Guba (bay) & $67^{\circ} 56^{\prime}$ & $52^{\circ} 46^{\prime}$ & $0 / 58$ & Tatarinov \& Kulakova (2013), Tatarinov (2016) \\
\hline Gorby (village) & $67^{\circ} 07^{\prime}$ & $44^{\circ} 30^{\prime}$ & $0 / 1$ & Poppius (1906) \\
\hline Jangech-Mylk (village) & $67^{\circ} 22^{\prime}$ & $55^{\circ} 52^{\prime}$ & $0 / 27$ & $\begin{array}{l}\text { Tatarinov \& Dolgin (1999), Tatarinov \& } \\
\text { Kulakova (2005), Tatarinov (2016) }\end{array}$ \\
\hline Kabanova (lake) & $66^{\circ} 18^{\prime}$ & $45^{\circ} 22^{\prime}$ & $0 / 2$ & Tatarinov (2016) \\
\hline Kambalnitsa (river) & $68^{\circ} 02^{\prime}$ & $44^{\circ} 26^{\prime}$ & $1 / 6$ & Poppius (1906), ZISP collection \\
\hline Kanin (peninsula) & - & - & $2 / 2$ & Hacker et al. (2002), MZH collection \\
\hline Kaninskij Kamen' (mountains) & $68^{\circ} 14^{\prime}$ & $44^{\circ} 51^{\prime}$ & $0 / 11$ & Tatarinov (2016) \\
\hline Kara (river) & $68^{\circ} 37^{\prime}$ & $65^{\circ} 13^{\prime}$ & $0 / 28$ & Tatarinov (2006a,b), Morgun (2017) \\
\hline Kharyaginskij (village) & $67^{\circ} 09^{\prime}$ & $56^{\circ} 43^{\prime}$ & $0 / 30$ & $\begin{array}{l}\text { Tatarinov \& Dolgin (1999), Tatarinov \& } \\
\text { Kulakova (2005), Tatarinov (2016) }\end{array}$ \\
\hline Khubtape (mount) & $69^{\circ} 12^{\prime}$ & $62^{\circ} 47^{\prime}$ & $0 / 1$ & Tatarinov (2006b) \\
\hline Kolokolkova Guba (bay) & $68^{\circ} 18^{\prime}$ & $52^{\circ} 32^{\prime}$ & $0 / 3$ & Tatarinov (2016) \\
\hline Korovinskaya Guba (bay) & $68^{\circ} 23^{\prime}$ & $53^{\circ} 27^{\prime}$ & $0 / 11$ & Tatarinov (2016) \\
\hline Krynka (river) & $68^{\circ} 32^{\prime}$ & $44^{\circ} 15^{\prime}$ & $0 / 12$ & Poppius (1906) \\
\hline Kuja (river) & $67^{\circ} 38^{\prime}$ & $53^{\circ} 17^{\prime}$ & $85 / 0$ & M.V.K. \& V.Z. leg. (2013) \\
\hline Kuznetskaya Guba (bay) & $68^{\circ} 51^{\prime}$ & $53^{\circ} 34^{\prime}$ & $0 / 4$ & Tatarinov (2016) \\
\hline Lovetskij (island) & $68^{\circ} 20^{\prime}$ & $53^{\circ} 55^{\prime}$ & $2 / 0$ & T. Dyaditsyna leg. (2016) \\
\hline Madakha (beacon) & $68^{\circ} 33^{\prime}$ & $44^{\circ} 45^{\prime}$ & $0 / 6$ & Poppius (1906) \\
\hline Malaya Padeya (mountains) & $69^{\circ} 04^{\prime}$ & $62^{\circ} 09^{\prime}$ & $0 / 16$ & $\begin{array}{l}\text { Tatarinov \& Gorbunov (2014), } \\
\text { Bolotov et al. (2015), Tatarinov (2016) }\end{array}$ \\
\hline Mikulkin (cape) & $67^{\circ} 49^{\prime}$ & $46^{\circ} 38^{\prime}$ & $0 / 8$ & Poppius (1906) \\
\hline More-Yu (river) & $68^{\circ} 19^{\prime}$ & $59^{\circ} 41^{\prime}$ & $0 / 3$ & Tatarinov (2016) \\
\hline Naryan-Mar (town) & $67^{\circ} 38^{\prime}$ & $53^{\circ} 00^{\prime}$ & $215 / 18$ & $\begin{array}{l}\text { M.V.K., V.Z. \& A. Stekolstchikov leg. } \\
\text { (2013, 2015), Tatarinov et al. (2003), } \\
\text { Tatarinov \& Kulakova (2005), } \\
\text { Tatarinov (2016) }\end{array}$ \\
\hline Nenetskaya Gryada (mountains) & $68^{\circ} 22^{\prime}$ & $53^{\circ} 06^{\prime}$ & $0 / 36$ & Tatarinov (2016) \\
\hline Nes' (village) & $66^{\circ} 35^{\prime}$ & $44^{\circ} 41^{\prime}$ & $0 / 33$ & Bolotov (2012), Tatarinov (2016) \\
\hline Padimey (lakes) & $67^{\circ} 35^{\prime}$ & $62^{\circ} 10^{\prime}$ & $0 / 27$ & Tatarinov \& Kulakova (2010), Tatarinov (2016) \\
\hline Pakhancheskaya Guba (bay) & $68^{\circ} 29^{\prime}$ & $57^{\circ} 13^{\prime}$ & $3 / 0$ & O. Makarova leg. (2015) \\
\hline Pechora site 2 (landmark) & $68^{\circ} 20^{\prime}$ & $53^{\circ} 05^{\prime}$ & $0 / 1$ & Ross (2000) \\
\hline Pechora site 3 (landmark) & $68^{\circ} 29^{\prime}$ & $53^{\circ} 54^{\prime}$ & $0 / 2$ & Ross (2000) \\
\hline Pechora site 4 (landmark) & $67^{\circ} 42^{\prime}$ & $53^{\circ} 07^{\prime}$ & $0 / 1$ & Ross (2000) \\
\hline Pechora site 7 (landmark) & $67^{\circ} 48^{\prime}$ & $54^{\circ} 01^{\prime}$ & $0 / 1$ & Ross (2000) \\
\hline Seida (river) & $67^{\circ} 24^{\prime}$ & $62^{\circ} 51^{\prime}$ & $0 / 54$ & Tatarinov (2016) \\
\hline Sengeiskij (strait) & $68^{\circ} 21^{\prime}$ & $51^{\circ} 22^{\prime}$ & $0 / 2$ & Tatarinov (2016) \\
\hline Shapkina (river), middle flow & $67^{\circ} 14^{\prime}$ & $54^{\circ} 39^{\prime}$ & $0 / 40$ & $\begin{array}{l}\text { Tatarinov \& Kulakova (2005), } \\
\text { Tatarinov (2006c, 2016) }\end{array}$ \\
\hline Shapkina (river), upper flow & $67^{\circ} 32^{\prime}$ & $54^{\circ} 56^{\prime}$ & $3 / 37$ & Tatarinov \& Kulakova (2005), Tatarinov (2016) \\
\hline Shoina (river) & $67^{\circ} 51^{\prime}$ & $44^{\circ} 10^{\prime}$ & $0 / 11$ & Bolotov (2012), Tatarinov (2016) \\
\hline Sibirchatayaha (river) & $68^{\circ} 44^{\prime}$ & $63^{\circ} 44^{\prime}$ & $0 / 28$ & Tatarinov (2016) \\
\hline Svetlaya (river) & $67^{\circ} 18^{\prime}$ & $49^{\circ} 26^{\prime}$ & $0 / 5$ & Tatarinov (2016) \\
\hline Syatorei (lake) & $67^{\circ} 28^{\prime}$ & $44^{\circ} 40^{\prime}$ & $0 / 11$ & Tatarinov (2016) \\
\hline Toshviska (village) & $67^{\circ} 07^{\prime}$ & $52^{\circ} 18^{\prime}$ & $49 / 0$ & T. Dyaditsyna leg. (2016) \\
\hline Ust-Kara (village) & $69^{\circ} 15^{\prime}$ & $64^{\circ} 55^{\prime}$ & $0 / 2$ & Tatarinov (2016) \\
\hline Varandey (village) & $68^{\circ} 49^{\prime}$ & $58^{\circ} 04^{\prime}$ & $0 / 5$ & Tatarinov (2016) \\
\hline Velikovisochnoye (village) & $67^{\circ} 15^{\prime}$ & $52^{\circ} 02^{\prime}$ & $4 / 3$ & $\begin{array}{l}\text { T. Dyaditsyna leg. (2014-2016), } \\
\text { Tatarinov (2016) }\end{array}$ \\
\hline Vesniyu (river) & $67^{\circ} 39^{\prime}$ & $54^{\circ} 43^{\prime}$ & $0 / 24$ & Tatarinov (2016) \\
\hline
\end{tabular}

1) Coordinates of some localities may have low accuracy (up to $50 \mathrm{~km}$ ) due to insufficient details provided in the original publications. This especially concerns localities associated with rivers and mountain ridges.

2) Before the slash: based on data that have not been published earlier; after the slash: based on published records. 
tersburg (ZISP) for specimens of all 108 species reported (Sinev 2008) from the biogeographical region that includes NAO. We also sought specimens from NAO in the so-called "Arctic collection" created by N. Y. Kusnezov in the 1930s and in accession materials deposited in ZISP. Finally, we carefully searched for published data on Lepidoptera from NAO and examined all published records for the reliability of identifications. We contacted the authors of the Catalogue of Lepidoptera of Russia (Sinev 2008) for clarification of those records for which we were unable to locate any supporting information. Note that, for the sake of brevity, we always refer to the entire catalogue, although the primary responsibility for the correctness of the information is held by the authors of each specific part of the catalogue.

\subsection{Nomenclature, data format and data analysis}

The nomenclature and the order of species generally follow the recent Nordic-Baltic checklist of Lepidoptera (Aarvik et al. 2017). An asterisk (*) indicates species that are recorded for the first time from NAO. An exclamation mark (!) indicates species that have been reported from this region by Sinev (2008) either erroneously or without a proper factual support, but have been discovered in NAO since then. Each record based on published data is referenced; an exception is butterflies for which the references are given only to excessively long lists of localities, as these are not included in the present manuscript. The abundance data are not included either, because this list is compilative and aims only at revealing species composition in the study region. Noteworthy species are provided with brief comments on their distribution and status. The similarities between the faunas of different regions were quantified by the Sørensen index (i.e. by the doubled number of common species divided by the sum of species numbers in two compared faunas, Sørensen 1948).

\section{Results}

\subsection{List of Lepidoptera species}

Eriocraniidae

* Eriocrania sp. Naryan-Mar. Larvae mining leaves of $B$. pubescens and Alnus alnobetula ssp. fruticosa. Barcoding (BOLD sequence pages: LRMNH067-17 and LRMNH068-17) surprisingly demonstrated that they belong to an undescribed species, which is close to $E$. sakhalinella Kozlov, described from Sakhalin Island in Russia and later found in Japan.

Hepialidae

* Hepialus humuli (Linnaeus). Kuja. Likely the northernmost record of this species.

Nepticulidae

* Stigmella lapponica (Wocke). Naryan-Mar.

* S. salicis (Stainton). Naryan-Mar.

* S. sorbi (Stainton). Naryan-Mar.

* Ectoedemia minimella (Zetterstedt). NaryanMar.

Prodoxidae

* Lampronia capitella (Clerck). Naryan-Mar.

* L. standfussiella Zeller. Toshviska. This species was considered rare for decades but appeared widely distributed in the northern regions, from Sweden to Labytnangi on the Asian side of the Ural Mts. (Kozlov et al. 2014).

* L. rupella (Denis \& Schiffermüller). Upper flow of Shapkina River.

Greya variabilis Davis \& Pellmyr. Amderma (Kullberg et al. 2013), Naryan-Mar. The record of this Beringian species in Naryan-Mar extends its distribution range $400 \mathrm{~km}$ to the west, indicating that $G$. variabilis can potentially be discovered in northern Fennoscandia. The moths were collected from several bogs around the city.

Incurvariidae

Incurvaria vetulella (Zetterstedt). Kambalnitsa (Poppius 1906), Madakha (Poppius 1906). The specimens have not been discovered in $\mathrm{MZH}$, so their identity remains problematic: they may actually represent both $I$. vetulella (Zetterstedt) and I. circulella (Zetterstedt).

* I. oehlmanniella (Hübner). Naryan-Mar. 
Adelidae

* Nemophora ochsenheimerella (Hübner). Naryan-Mar. This very unexpected finding of a species feeding on conifers narrows the distribution gap between Denmark (Aarvik et al. 2017) and Asia, where this species is known from Eastern Siberia to Japan (Kozlov 1997).

Tineidae

Tineola bisselliella (Hummel). Amderma (Kullberg et al. 2013).

Gracillariidae

* Parornix loganella (Stainton). Naryan-Mar.

* P. polygrammella (Wocke). Naryan-Mar.

* Phyllonorycter ulmifoliella (Hübner). NaryanMar.

* P. sorbi (Frey). Naryan-Mar.

* P. hilarella (Zetterstedt). Naryan-Mar.

Yponomeutidae

* Paraswammerdamia lapponica (W. Petersen). Naryan-Mar.

* P. conspersella (Tengström). Naryan-Mar.

Argyresthiidae

* Argyresthia laevigatella (Heydenreich). Naryan-Mar.

* A. glabratella (Zeller). Naryan-Mar.

* A. svenssoni Bengtsson \& Johansson. NaryanMar. This recently described species has so far been known from Sweden, Norway, Finland, Italy, Slovakia and Switzerland (Lewis \& Sohn 2015, Schmid 2016). The first record from Russia shows that $A$. svenssoni has a much wider distribution that could be originally expected, so it may well be found in Asia as well.

* A. abdominalis Zeller. Naryan-Mar.

* A. pygmaeella (Denis \& Schiffermüller). Kuja, Naryan-Mar.

* A. conjugella Zeller. Naryan-Mar.

Plutellidae

Plutella xylostella (Linnaeus). Amderma (Kullberg et al. 2013), Bugryanitsa (Poppius 1906), Chizha (Poppius 1906), Gorby (Poppius 1906), Krynka (Poppius 1906), Kuja, Lovetskij, Naryan-Mar, Toshviska.

* P. porrectella (Linnaeus). Kuja, Naryan-Mar.
Glyphipterigidae

* Glyphipterix forsterella (Fabricius). NaryanMar.

* G. simpliciella (Stephens). Kuja, Naryan-Mar.

Ypsolophidae

* Ypsolopha scabrella (Linnaeus). Naryan-Mar.

Choreutidae

* Anthophila fabriciana (Linnaeus). NaryanMar.

Tortricidae

* Sparganothis rubicundana (Herrich-Schäffer). Kuja, Naryan-Mar.

* Clepsis rogana (Guenée). Kuja, Naryan-Mar.

* C. moeschleriana (Wocke). Naryan-Mar. The westernmost record of this Beringian species, which earlier was reported from Taymyr Peninsula (Kozlov et al. 2006) and Polar Ural (Aarvik 2013).

! Aphelia viburnana (Denis \& Schiffermüller). Kuja, Naryan-Mar.

* Lozotaenia forsterana (Fabricius). NaryanMar.

* Syndemis musculana (Hübner). Naryan-Mar, Toshviska.

! Eana osseana (Scopoli). Bugryanitsa (Poppius 1906: as Cnephasia longana), Kuja, NaryanMar, Toshviska.

* Acleris comariana (Lienig \& Zeller). NaryanMar, Toshviska.

! A. effractana (Hübner). Toshviska. Earlier this species was indicated from this region as $A$. emargana (Sinev 2008).

! A. maccana (Treitschke). Kanin Peninsula.

* A. implexana (Walker). Naryan-Mar.

* Eulia ministrana (Linnaeus). Kuja, NaryanMar.

* Aethes smeathmanniana (Fabricius). Kuja, Naryan-Mar.

* Cochylidia subroseana (Haworth.). NaryanMar.

* C. implicitana (Wocke). Naryan-Mar.

* Pseudosciaphila branderiana (Linnaeus). Kuja.

Apotomis betuletana (Haworth). Naryan-Mar.

A. frigidana (Packard). Amderma (Kullberg et al. 2013).

* Orthotaenia undulana (Denis \& Schiffermüller). Naryan-Mar. 
Celypha lacunana (Denis \& Schiffermüller). Kuja, Naryan-Mar.

* Phiaris umbrosana (Freyer). Kuja, NaryanMar.

! P. obsoletana (Zetterstedt). Amderma (Kullberg et al. 2013), Kanin Peninsula, Kuja, NaryanMar, upper flow of Shapkina River, Toshviska.

P. metallicana (Hübner). Krynka (Poppius 1906), Mikulkin (Poppius 1906).

* P. schulziana (Fabricius). Kambalnitsa (Poppius 1906), Madakha (Poppius 1906).

! P. turfosana (Herrich-Schäffer). Amderma (Kullberg et al. 2013), Kuja, Naryan-Mar.

* P. palustrana (Lienig \& Zeller). Naryan-Mar, Toshviska.

* P. septentrionana (Curtis). Kuja, Naryan-Mar.

* P. bipunctana (Fabricius). Kuja, Naryan-Mar.

P. inquietana (Walker). Amderma (Kullberg et al. 2013). A locally abundant Beringian species, which lives on Pedicularis oederi Wahl. (Kuznetsov 1978). The foodplant has a gap in distribution between Scandinavian mountain regions and northern Russia (Hultén 1986), which may explain the absence of this species in northern Fennoscandia.

* Argyroploce lediana (Linnaeus). Naryan-Mar.

A. noricana (Herrich-Schäffer). Amderma (Kullberg et al. 2013).

* Bactra lancealana (Hübner). Kuja, NaryanMar.

* Ancylis unguicella (Linnaeus). Kuja, NaryanMar, Toshviska.

* A. subarcuana (Douglas). Kuja, Naryan-Mar.

* A. habeleri Huemer \& Tarmann. Kuja. This species, described from Austria, was since then found from France (Blanchemain 2015) to Iran (Alipanah 2015) and Finland (Aarvik et al. 2017). The first record from Russia substantially expands its distribution range to the North and East.

* A. badiana (Denis \& Schiffermüller). Kuja, Naryan-Mar.

Eriopsela quadrana (Hübner). Kambalnitsa (Poppius 1906), Madakha (Poppius 1906).

* Epinotia immundana (Fischer von Röslerstamm). Kuja, Naryan-Mar.

* E. tetraquetrana (Haworth). Kuja, NaryanMar.
* E. nemorivaga (Tengström). Kuja, NaryanMar.

* E. tedella (Clerck). Naryan-Mar.

* E. granitana (Herrich-Schäffer). Naryan-Mar.

* E. cruciana (Linnaeus). Kuja, Naryan-Mar.

* E. gimmerthaliana (Lienig \& Zeller). NaryanMar.

* E. nanana (Treitschke). Naryan-Mar.

! E. crenana (Hübner). Toshviska.

* Pelochrista guentheri (Tengström). NaryanMar.

* Eucosma obumbratana (Lienig \& Zeller). Naryan-Mar.

* E. aspidiscana (Hübner). Naryan-Mar.

* E. suomiana (A. Hoffmann). Kuja.

E. ommatoptera Falkovich. Amderma (Kullberg et al. 2013).

* E. tetraplana (Möschler). Naryan-Mar. This species is widely distributed in Russia (Sinev 2008) but does not occur in the Nordic and Baltic countries (Aarvik et al. 2017).

Gypsonoma parryana (Curtis). Amderma (Kullberg et al. 2013).

Epiblema scutulana (Denis \& Schiffermüller). Mikulkin (Poppius 1906).

* Dichrorampha plumbana (Scopoli). Kuja, Naryan-Mar.

* D. sedatana Busck. Kuja, Naryan-Mar.

* D. acuminatana (Lienig \& Zeller). NaryanMar.

* D. montanana (Duponchel). Kuja, NaryanMar. The northernmost records of this species, which does not occur in the Nordic and Baltic countries (Aarvik et al. 2017).

* D. plumbagana (Treitschke). Naryan-Mar.

* Grapholita compositella (Fabricius). NaryanMar.

* G. orobana Treitschke. Kuja, Naryan-Mar.

* Pammene clanculana (Tengström). NaryanMar.

* P. obscurana (Stephens). Naryan-Mar.

* P. aurana (Fabricius). Naryan-Mar.

Oecophoridae

* Denisia stroemella (Fabricius). Naryan-Mar.

! Pleurota bicostella (Clerck). Naryan-Mar.

* Ethmia quadrillella (Goeze). Naryan-Mar. 
Gelechiidae

* Neofaculta infernella (Herrich-Schäffer). Kuja, Naryan-Mar.

* Acompsia subpunctella Svensson. Kuja, Naryan-Mar.

Bryotropha galbanella (Zeller). Amderma (Kullberg et al. 2013).

* Monochroa sp. nr. simplicella (Lienig \& Zeller). Naryan-Mar. This species, which has also been collected from Polar Ural by J. Jalava and J. Kullberg, is now under description by O. Karsholt.

* Athrips pruinosella (Lienig \& Zeller). NaryanMar.

Prolita sexpunctella (Fabricius). Krynka (Poppius 1906), Madakha (Poppius 1906), Naryan-Mar.

* Chionodes continuella (Zeller). Naryan-Mar.

* C. viduella (Fabricius). Naryan-Mar.

Gnorimoschema vastificum Braun. Amderma (Kullberg et al. 2013).

Elachistidae

* Elachista subalbidella Schläger. Naryan-Mar.

* E. eleochariella Stainton. Naryan-Mar.

* E. alpinella Stainton. Kuja, Naryan-Mar.

* E. diederichsiella E. Hering. Kuja, NaryanMar.

* E. zernyi Hartig. Naryan-Mar.

* E. apicipunctella Stainton. Kuja, Naryan-Mar.

* E. maculicerusella Bruand. Naryan-Mar.

* E. baltica E. Hering. Naryan-Mar.

Coleophoridae

* Coleophora striatipennella Nylander. NaryanMar.

* C. trochilella (Duponchel). Naryan-Mar.

* C. squamosella Stainton. Naryan-Mar.

* C. virgaureae Stainton. Kuja, Naryan-Mar.

* C. pappiferella O. Hofmann. Naryan-Mar.

* C. alticolella Zeller. Kuja, Naryan-Mar.

* C. frischella (Linnaeus). Kuja, Naryan-Mar.

* C. alcyonipennella (Kollar). Kuja, NaryanMar.

* C. idaeella O. Hofmann. Naryan-Mar.

* C. ledi Stainton. Naryan-Mar.

* C. plumbella Kanerva. Kuja, Naryan-Mar.

* C. glitzella O. Hofmann. Naryan-Mar.

* C. murinella Tengström. Naryan-Mar.
* C. albidella (Denis \& Schiffermüller). NaryanMar.

* C. sp. Naryan-Mar. This species, which does not resemble any known representative of the Palaearctic fauna, is now being examined by J. Tabell.

Momphidae

* Mompha raschkiella (Zeller). Naryan-Mar.

* M. conturbatella (Hübner). Naryan-Mar.

Scythrididae

* Scythris obscurella (Scopoli). Naryan-Mar.

* S. noricella (Zeller). Naryan-Mar.

* S. fuscopterella Bengtsson. Naryan-Mar.

Epermeniidae

* Phaulernis fulviguttella (Zeller). Kuja, NaryanMar, Toshviska.

Pterophoridae

* Platyptilia gonodactyla (Denis \& Schiffermüller). Naryan-Mar.

P. calodactyla (Denis \& Schiffermüller). Amderma (Kullberg et al. 2013), Kuja, NaryanMar.

* Gillmeria pallidactyla (Haworth). Kuja, Naryan-Mar.

Paraplatyptilia sahlbergi (Poppius). Krynka (Poppius 1906).

P. sibirica (Zagulajev). Amderma (Kullberg et al. 2013).

* Stenoptilia pterodactyla (Linnaeus). Kuja, Naryan-Mar.

* S. veronicae Karvonen. Kuja, Naryan-Mar.

* Hellinsia didactylites (Ström). Naryan-Mar.

Papilionidae

Parnassius corybas Fischer de Waldheim (phoebus auct. nec Fabricius). Kara; several more localities in the eastern part of NAO were listed by Tatarinov (2006a). The westernmost records of this Siberian species.

Papilio machaon Linnaeus. Golodnaya Guba, Kharyaginskij, Kuja, Malaya Padeya, Naryan-Mar, middle flow of Shapkina River, and other 13 localities (Tatarinov 2016).

Hesperiidae

Pyrgus centaureae (Rambur). Golodnaya Guba, 
Jangech-Mylk, Kara, Kharyaginskij, Nes', Padimey Lakes, and other six localities (Tatarinov 2016).

Carterocephalus palaemon (Pallas). Golodnaya Guba. Kuja, Naryan-Mar, Padimey Lakes, Toshviska, and other eight localities (Tatarinov 2016).

C. silvicola (Meigen). Nenetskaya Gryada, Golognaya Guba, Kuja, Naryan-Mar, Seyda.

Hesperia comma (Linnaeus). Padimey Lakes, upper flow of Shapkina River.

Pieridae

Leptidea sinapis (Linnaeus). Naryan-Mar, Nes', middle flow of Shapkina River, Seyda.

Anthocharis cardamines (Linnaeus). JangechMylk, Kharyaginskij, Naryan-Mar, Nes', Padimey Lakes, upper flow of Shapkina River, and other eight localities (Tatarinov 2016).

Aporia crataegi (Linnaeus). Amderma, Golodnaya Guba, Kharyaginskij, NaryanMar, Nes', and other seven localities (Tatarinov 2016).

Pieris rapae (Linnaeus). Kharyaginskij, NaryanMar, Nes', Toshviska, Velikovisochnoe, and other five localities (Tatarinov 2016).

P. napi (Linnaeus). Amderma, Golodnaya Guba, Kara (Morgun 2017 as P. bryoniae), Kuja, Naryan-Mar, Pechora site 4, Toshviska, and other 15 localities (Tatarinov 2016).

Pontia daplidice (Linnaeus). Sibirchatayaha (as P. callidice: Tatarinov 2016).

Colias hyale (Linnaeus). Middle flow of Shapkina River, Seyda.

C. hecla Lefèbvre. Chernaya, Jangech-Mylk, Kara, Malaya Padeya, Padimey Lakes, middle flow of Shapkina River, Sibirchatayaha.

C. tyche (Böber). Kara, Khubtape, Sibirchatayaha.

C. palaeno (Linnaeus). Golodnaya Guba, Jangech-Mylk, Kara, Naryan-Mar, Nes', Toshviska, and other 17 localities (Tatarinov 2016).

Gonepteryx rhamni (Linnaeus). Naryan-Mar, Nes', upper flow of Shapkina River, Kabanova.

Nymphalidae

Boloria eunomia (Esper). Golodnaya Guba,
Jangech-Mylk, Kara, Kharyaginskij, NaryanMar, Nes', and other eight localities (Tatarinov 2016).

B. euphrosyne (Linnaeus). Naryan-Mar, Nes', middle flow of Shapkina River, Seyda.

B. selene (Denis \& Schiffermüller). Golodnaya Guba, Jangech-Mylk, Kara, Kharyaginskij, Kuja, Naryan-Mar, and other eight localities (Tatarinov 2016).

B. chariclea (Schneider). Amderma, Golodnaya Guba, Jangech-Mylk, Kara, Malaya Padeya, Seyda, and other four localities (Tatarinov 2016).

B. freija (Thunberg). Golodnaya Guba. JangechMylk, Kharyaginskij, Malaya Padeya, Naryan-Mar, and other 13 localities (Tatarinov 2016).

B. polaris (Boisduval). Kara, Malaya Padeya, upper flow of Shapkina River, Ust-Kara.

B. thore (Hübner). Golodnaya Guba, JangechMylk, Naryan-Mar, Padimey Lakes, upper flow of Shapkina River, and other five localities (Tatarinov 2016).

B. frigga (Thunberg). Amderma, Golodnaya Guba, Jangech-Mylk, Kara, Kharyaginskij, Naryan-Mar, and other 14 localities (Tatarinov 2016).

B. improba (Butler). Amderma, Jangech-Mylk, Kara, Kuznetskaya, Malaya Padeya, Varandey, and other five localities (Tatarinov 2016).

B. aquilonaris (Stichel). Golodnaya Guba, Jangech-Mylk, Kharyaginskij, Kuja, NaryanMar, and other ten localities (Tatarinov 2016).

B. alaskensis (Holland). Amderma, Chernaya, Golodnaya Guba, Jangech-Mylk, Kara, Malaya Padeya, Nenetskaya Gryada.

B. angarensis (Erschoff). Golodnaya Guba, Naryan-Mar, Padimey Lakes, Seyda, upper flow of Shapkina River, Vesniju.

Issoria eugenia (Eversmann). Kara, Kharyaginskij, Padimey Lakes, Seyda, upper and middle flow of Shapkina River, Sibirchatayaha.

Brenthis ino (Rottemburg). Kuja, Naryan-Mar, middle flow of Shapkina River, Seyda.

Argynnis aglaja (Linnaeus). Kuja, Naryan-Mar, middle flow of Shapkina River, Seyda.

Araschnia levana (Linnaeus). Nes', middle flow of Shapkina River, Seida. 
Vanessa atalanta (Linnaeus). Naryan-Mar, Seida, upper flow of Shapkina River, Velikovisochnoe. Occasional migrant.

V. cardui (Linnaeus). Krynka, Malaya Padeya, Naryan-Mar, Pechora site 3, Toshviska, Velikovisochnoe, and other 11 localities (Tatarinov 2016). Occasional migrant.

Aglais urticae (Linnaeus). Kharyaginskij, Naryan-Mar, Nenetskaya Gryada, Nes', Seida.

Nymphalis antiopa (Linnaeus). Amderma, Golodnaya Guba, Kharyaginskij, Naryan-Mar, Nenetskaya Gryada, Nes', Seida, upper and middle flow of Shapkina River.

N. xanthomelas (Esper). Amderma, Golodnaya Guba, Kara, Malaya Padeya, Naryan-Mar, Padimey Lakes, upper and middle flow of Shapkina River, Toshviska, and other nine localities (Tatarinov 2016).

Polygonia c-album (Linnaeus). Seida, middle flow of Shapkina River.

Euphydryas iduna (Dalman). Jangech-Mylk, Naryan-Mar, Nenetskaya Gryada, upper flow of Shapkina River.

E. maturna (Linnaeus). Naryan-Mar, Seida, middle flow of Shapkina River.

Melitaea athalia (Rottemburg). Kabanova.

Coenonympha tullia (Müller). Golodnaya Guba. Jangech-Mylk, Kara, Kharyaginskij, Kuja, Naryan-Mar, Padimey Lakes, upper and middle flow of Shapkina River, and other 11 localities (Tatarinov 2016).

Erebia ligea (Linnaeus). Jangech-Mylk, Seida, middle flow of Shapkina River.

E. euryale (Esper). Golodnaya Guba, Kaninskij Kamen', Kharyaginskij, Kuja, Naryan-Mar, Nenetskaya Gryada, Nes', Seida, Shoina, middle flow of Shapkina River.

E. embla (Thunberg). Naryan-Mar, Seida. The record from Kharyaginskij (Tatarinov and Dolgin 1999) is based on a mislabelled specimen (A. Tatarinov, pers. comm.).

E. disa (Thunberg). Golodnaya Guba, JangechMylk, Kharyaginskij, Malaya Padeya, Naryan-Mar, Nes', Padimey Lakes, upper and middle flow of Shapkina River, and other nine localities (Tatarinov 2016).

E. pandrose (Borkhausen). Kambalnitsa, Kaninskij Kamen', Korovinskaya, Krynka, Mikulkin, Naryan-Mar, Shoina.
E. discoidalis (Kirby). Kara, Kharyaginskij, Seida.

E. fasciata Butler. Golodnaya Guba, JangechMylk, Kara, Kharyaginskij, Malaya Padeya, Naryan-Mar, Padimey Lakes, upper and middle flow of Shapkina River, and other seven localities (Tatarinov 2016)

E. jeniseiensis Trybom. Golodnaya Guba, Jangech-Mylk, Nenetskaya Gryada, NaryanMar, Padimey Lakes, upper flow of Shapkina River, Vesniju.

E. rossii (Curtis). Jangech-Mylk, Kharyaginskij, Malaya Padeya, Naryan-Mar, Padimey Lakes, upper and middle flow of Shapkina River, and other 14 localities (Tatarinov 2016).

Oeneis norna (Thunberg). Golodnaya Guba, Jangech-Mylk, Kharyaginskij, Naryan-Mar, Nenetskaya Gryada, Nes', Padimey Lakes, Seida, upper and middle flow of Shapkina River, Velikovisochnoe, Vesniju.

O. bore (Schneider). Jangech-Mylk, Kharyaginskij, Naryan-Mar, Padimey Lakes, upper and middle flow of Shapkina River, and other six localities (Tatarinov 2016).

O. jutta (Hübner). Kharyaginskij, Nes', Seida, middle flow of Shapkina River.

O. melissa (Fabricius). Kara, Sibirchatayaha.

Lycaenidae

Cupido minimus (Fuessly). Golodnaya Guba, Nenetskaya Gryana, Naryan-Mar, Seida.

Celastrina argiolus (Linnaeus). Kharyaginskij, Naryan-Mar, Seida, upper and middle flow of Shapkina River.

Plebeius idas (Linnaeus). Golodnaya Guba, Naryan-Mar, Nes', Seida, upper and middle flow of Shapkina River.

Aricia artaxerxes (Fabricius). Golodnaya Guba, Naryan-Mar, Nes', middle flow of Shapkina River, Seida.

Eumedonia eumedon (Esper). Golodnaya Guba, Naryan-Mar, Seida.

Cyaniris semiargus (Rottemburg). Golodnaya Guba, Kharyaginskij, Kuja, Naryan-Mar, Nenetskaya Gryada, Nes', Seida, Toshviska. Agriades optilete (Knoch). Golodnaya Guba, Jangech-Mylk, Kara, Kharyaginskij, Kuja, Malaya Padeya, Naryan-Mar, Nes', Padimey Lakes, Shoina, upper and middle flow of 
Shapkina River, and other ten localities (Tatarinov 2016).

A. aquilo (Boisduval). Kara, Sibirchatayaha (as A. glandon; Tatarinov 2016).

Polyommatus icarus (Rottemburg). Kharyaginskij, Kuja, Naryan-Mar, Nes', Vesniju, Seida, middle flow of Shapkina River.

P. eros (Ochsenheimer). Kara, Seida.

Lycaena phlaeas (Linnaeus). Kharyaginskij, Naryan-Mar, Seida, upper flow of Shapkina River.

Lycaena helle (Denis \& Schiffermüller). Golodnaya Guba, Kuja, Naryan-Mar, Nenetskaya Gryada, Nes', Padimey Lakes, Pechora site 2, upper and middle flow of Shapkina River, Vesniju.

Lycaena hippothoe (Linnaeus). Golodnaya Guba, Kharyaginskij, Naryan-Mar, Nenetskaya Gryada, Padimey Lakes, Seida, upper flow of Shapkina River, Vesniju.

Callophrys rubi (Linnaeus). Golodnaya Guba, Kharyaginskij, Lovetskij, Naryan-Mar, Nenetskaya Gryada, Nes', Seida, Padimey Lakes, Pechora site 7, upper and middle flow of Shapkina River.

Pyralidae

* Anerastia lotella (Hübner). Kuja, Naryan-Mar.

* Catastria marginea (Denis \& Schiffermüller). Naryan-Mar.

C. kistrandella Opheim. Amderma (Kullberg et al. 2013).

Pyla fusca (Haworth). Krynka (Poppius 1906), Kuja, Mikulkin (Poppius 1906), Naryan-Mar. Polopeustis altensis (Wocke). Krynka (Poppius 1906), Mikulkin (Poppius 1906).

* Anania funebris (Ström). Kuja, Naryan-Mar.

* A. fuscalis (Denis \& Schiffermüller). Toshviska.

* Udea inquinatalis (Lienig \& Zeller). Kuja, Naryan-Mar.

U. decrepitalis (Herrich-Schäffer). Krynka (Poppius 1906), Kuja, Naryan-Mar.

U. alaskalis (Gibson). Amderma (Kullberg et al. 2013).

U. uralica Slamka. Amderma (Kullberg et al. 2013), Kambalnitsa, Kuja, Naryan-Mar. This species, which was recently described from Ural Mts., seems to be widespread in northern
Russia, and our new records expand its distribution range some $800 \mathrm{~km}$ to the West. The earlier record of $U$. cretacea Fil. from Taymyr (Kozlov et al. 2006) should be attributed to this species.

Eudonia alpina (Curtis). Kambalnitsa (Poppius 1906), Madakha (Poppius 1906), NaryanMar.

* E. murana (Curtis). Naryan-Mar.

Catoptria furcatellus (Zetterstedt). Bugryanitsa (Poppius 1906).

* C. maculalis (Zetterstedt). Kuja, Naryan-Mar.

Pediasia zellerella (Staudinger). Amderma (Kullberg et al. 2013).

* Chrysoteuchia culmella (Linnaeus). Kuja, Naryan-Mar.

* Crambus pascuella (Linnaeus). Naryan-Mar.

* C. silvella (Hübner). Naryan-Mar.

* C. ericella (Hübner). Naryan-Mar.

* C. alienellus (Germar \& Kaulfuss). NaryanMar.

* C. hamella (Thunberg). Kuja, Naryan-Mar.

* C. perlella (Scopoli). Kuja, Naryan-Mar.

Drepanidae

* Ochropacha duplaris (Linnaeus). Toshviska.

Geometridae

Cyclophora pendularia (Clerck). Golodnaya Guba (Tatarinov \& Kulakova 2013).

* Timandra comae A. Schmidt. Naryan-Mar.

* T. griseata W. Petersen. Naryan-Mar.

* Scopula frigidaria (Möschler). Kuja, NaryanMar.

S. ternata Schrank. Golodnaya Guba (Tatarinov \& Kulakova 2013), Naryan-Mar.

Scotopteryx chenopodiata (Linnaeus).

Golodnaya Guba (Tatarinov \& Kulakova 2013).

Psychophora sabini Kirby. Amderma (Kullberg et al. 2013).

* Xanthorhoe designata (Hufnagel). NaryanMar, Toshviska.

* X. abrasaria (Herrich-Schäffer). Kuja, NaryanMar.

* X. decoloraria (Esper). Kuja, Naryan-Mar, Toshviska.

* X. spadicearia (Denis \& Schiffermüller). Naryan-Mar.

* X. ferrugata (Clerck). Naryan-Mar, Toshviska. 
* X. montanata (Denis \& Schiffermüller). Kuja, Naryan-Mar, Toshviska.

* X. fluctuata (Linnaeus). Toshviska.

$X$. annotinata (Zetterstedt). Krynka (Poppius 1906), Madakha (Poppius 1906), NaryanMar, Toshviska.

X. uralensis Choi. Amderma (Kullberg et al. 2013).

* Epirrhoe hastulata (Hübner). Kuja, NaryanMar.

* E. alternata (Müller). Naryan-Mar, Toshviska.

Entephria polata (Duponchel). Pakhancheskaya Guba, Toshviska

E. byssata (Aurivillius). Amderma (Kullberg et al. 2013, as E. punctipes), Kambalnitsa (Poppius 1906), Krynka (Poppius 1906), Mikulkin (Poppius 1906), Pakhancheskaya Guba.

E. caesiata (Denis \& Schiffermüller). NaryanMar.

* Spargania luctuata (Denis \& Schiffermüller). Kuja, Naryan-Mar.

* Hydriomena furcata (Thunberg). Bolvanskaya Guba, Naryan-Mar, Toshviska.

* H. impluviata (Denis \& Schiffermüller). Naryan-Mar, Toshviska.

* Heterothera serraria (Lienig \& Zeller). Naryan-Mar.

* Eulithis testata (Linnaeus). Toshviska.

! E. populata (Linnaeus). Kuja, Naryan-Mar, Toshviska.

* Dysstroma citrata (Linnaeus). Toshviska.

D. pseudimmanata (Heydenreich). Amderma (Kullberg et al. 2013).

* Colostygia turbata (Hübner). Naryan-Mar.

* Lampropteryx otregiata (Metcalfe). Toshviska.

* Epirrita autumnata (Borkhausen). Toshviska, Velikovisochnoe.

! Rheumaptera hastata (Linnaeus). Golodnaya Guba (Tatarinov \& Kulakova 2013), Kuja, Naryan-Mar.

$R$. subhastata (Nolcken). Golodnaya Guba (Tatarinov \& Kulakova 2013), Naryan-Mar.

* Coenocalpe lapidata (Hübner). Toshviska.

* Perizoma affinitata (Stephens). Toshviska.

* P. alchemillata (Linnaeus). Toshviska.

* P. blandiata (Denis \& Schiffermüller). Kuja, Naryan-Mar, Toshviska.

* P. albulata (Denis \& Schiffermüller). NaryanMar.
* Pasiphila debiliata (Hübner). Upper flow of Shapkina River.

* Eupithecia abietaria (Goeze). Naryan-Mar.

* E. pygmaeata (Hübner). Kuja, Naryan-Mar.

* E. gelidata Möschler. Naryan-Mar.

* E. conterminata (Lienig \& Zeller). NaryanMar.

* E. veratraria Herrich-Schäffer Kuja, NaryanMar.

* E. intricata (Zetterstedt). Naryan-Mar.

! E. satyrata (Hübner). Kuja.

* E. vulgata (Haworth). Naryan-Mar.

* E. succenturiata (Linnaeus). Kuja, NaryanMar, Toshviska.

! Carsia sororiata (Hübner). Naryan-Mar, Toshviska.

* Macaria alternata (Denis \& Schiffermüller). Kuja, Naryan-Mar, Toshviska.

M. brunneata (Thunberg). Mikulkin (Poppius 1906), Naryan-Mar.

M. fusca (Thunberg). Bugryanitsa (Poppius 1906), Naryan-Mar, Pakhancheskaya Guba.

* Chiasmia clathrata (Linnaeus). Kuja, NaryanMar, Toshviska.

Ematurga atomaria (Linnaeus). Golodnaya Guba (Tatarinov \& Kulakova 2013).

Ennomos autumnaria (Werneburg). Golodnaya Guba (Tatarinov \& Kulakova 2013).

Elophos vittaria (Thunberg). Krynka (Poppius 1906), Naryan-Mar.

Glacies coracina (Esper). Golodnaya Guba (Tatarinov \& Kulakova 2013).

Saturniidae

Saturnia pavonia (Linnaeus). Golodnaya Guba (Tatarinov \& Kulakova 2013); middle flow of Shapkina River (Tatarinov 2006c).

Sphingidae

Hyles gallii (Rottemburg). Golodnaya Guba (Tatarinov \& Kulakova 2013), Kharyaginskij (Tatarinov et al. 2003), Naryan-Mar (Tatarinov et al. 2003).

Erebidae

Scoliopteryx libatrix (Linnaeus). Golodnaya Guba (Tatarinov \& Kulakova 2013).

Apantesis quenseli (Paykull). Golodnaya Guba

(Tatarinov \& Kulakova 2013).

Arctia lapponica (Thunberg). Golodnaya Guba

(Tatarinov \& Kulakova 2013). 
A. subnebulosa tundrana Tshistjakov. Amderma (Kullberg et al. 2013), Malaya Padeya (Bolotov et al. 2015).

Euclidia glyphica (Linnaeus). Golodnaya Guba (Tatarinov \& Kulakova 2013), Naryan-Mar.

* E. mi (Clerck). Naryan-Mar.

Noctuidae

Diachrysia chrysitis (Linnaeus). Golodnaya Guba (Tatarinov \& Kulakova 2013).

* D. stenochrysis (Warren). Toshviska.

Autographa gamma (Linnaeus). Golodnaya Guba (Tatarinov \& Kulakova 2013), Pechora site 3 (Ross 2000), Toshviska.

Syngrapha hochenwarthi (Hochenwarth). Amderma (Kullberg et al. 2013), Kuja, Naryan-Mar.

* S. microgamma (Hübner). Naryan-Mar.

Sympistis zetterstedtii (Staudinger). Amderma (Kullberg et al. 2013), Golodnaya Guba (Tatarinov \& Kulakova 2013).

* Helicoverpa armigera (Hübner). Toshviska. A subtropical migrant species, which is regularly recorded in the Nordic and Baltic countries (Aarvik et al. 2017).

* Amphipoea fucosa (Freyer). Toshviska.

* Hillia iris (Zetterstedt). Toshviska.

Coranarta cordigera (Thunberg) Golodnaya Guba (Tatarinov \& Kulakova 2013).

Polia conspicua (A. Bang-Haas). Kanin Pns. (Hacker et al. 2002: distribution map).

Polia richardsoni (Curtis). Amderma (Kullberg et al. 2013), Kanin Pns. (Hacker et al. 2002: distribution map).

* Papestra biren (Goeze). Naryan-Mar.

Agrotis exclamationis (Linnaeus). Golodnaya

Guba (Tatarinov \& Kulakova 2013).

* Diarsia mendica (Fabricius). Naryan-Mar.

Eurois occulta (Linnaeus). Golodnaya Guba (Tatarinov \& Kulakova 2013), Toshviska.

Xestia quieta (Hübner). Amderma (Kullberg et al. 2013), Mikulkin (Poppius 1906).

$X$. liquidaria (Eversmann). Amderma (Kullberg et al. 2013).

\subsection{Species excluded from the list}

In total, 30 species has been erroneously reported, or reported without a proper factual support, from
NAO and the Novaya Zemlya region in the Catalogue of the Lepidoptera of Russia (Sinev 2008). However, 12 of these species (labelled with an exclamation mark in the list above) have been found to occur in NAO since then, so only the records of the following 18 species have not yet been confirmed.

Choreutis diana (Hübner), Phiaris turfosana (Herrich-Schäffer), Epinotia crenana (Hübner) and Macaria carbonaria (Clerck) were included in the Catalogue based on misinterpreted records by Poppius (1906), who recorded these species for a locality that lies outside NAO (S. Sinev \& V. Mironov, pers. comm.). Similarly, Malacodea regelaria Tengström was reported from the region based on the specimen from Ust-Tsilma, Republic of Komi (V. Mironov, pers. comm.).

Three species were reported based on misidentified specimens. Cnephasia longana (Haworth) from Bugryanitsa (Poppius 1906) appeared to be Eana osseana (Scopoli), Epagoge grotiana (Fabricius) from Dolgij Island appeared to be Clepsis mehli (Opheim) and Apotomis demissana Kennel from Dolgij Island was reidentified as Apotomis frigidana (Packard).

The authors of the Catalogue were unable to provide any information regarding the reasons for reporting the following species from the region that includes NAO and Novaya Zemlya archipelago: Rhigognostis senilella (Zetterstedt), Acleris hastiana (Linnaeus), Apotomis sororculana (Zetterstedt), Phiaris glaciana (Möschl.), Gesneria centuriella (Denis \& Schiffermüller), Loxostege sticticalis (Linnaeus), Gynaephora relictus (O. Bang-Haas), Leucoma salicis (Linnaeus), Polia lamuta (Herz) and Xestia tecta (Hübner) (S. Sinev and A. Matov, pers. comm.). All these species were therefore excluded from our list.

Parnassius mnemosyne (Linnaeus) was reported from NAO by Bolotov et al. (2012) based on the record made near Kosminskoe Lake, which is located at the border of NAO, Arkhangelsk oblast and the Komi Republic. However, the species was collected on the territory of the Komi Republic (Tatarinov 2016), so its occurrence in NAO, although very likely, remains to be confirmed (A. Tatarinov, pers. comm.). 


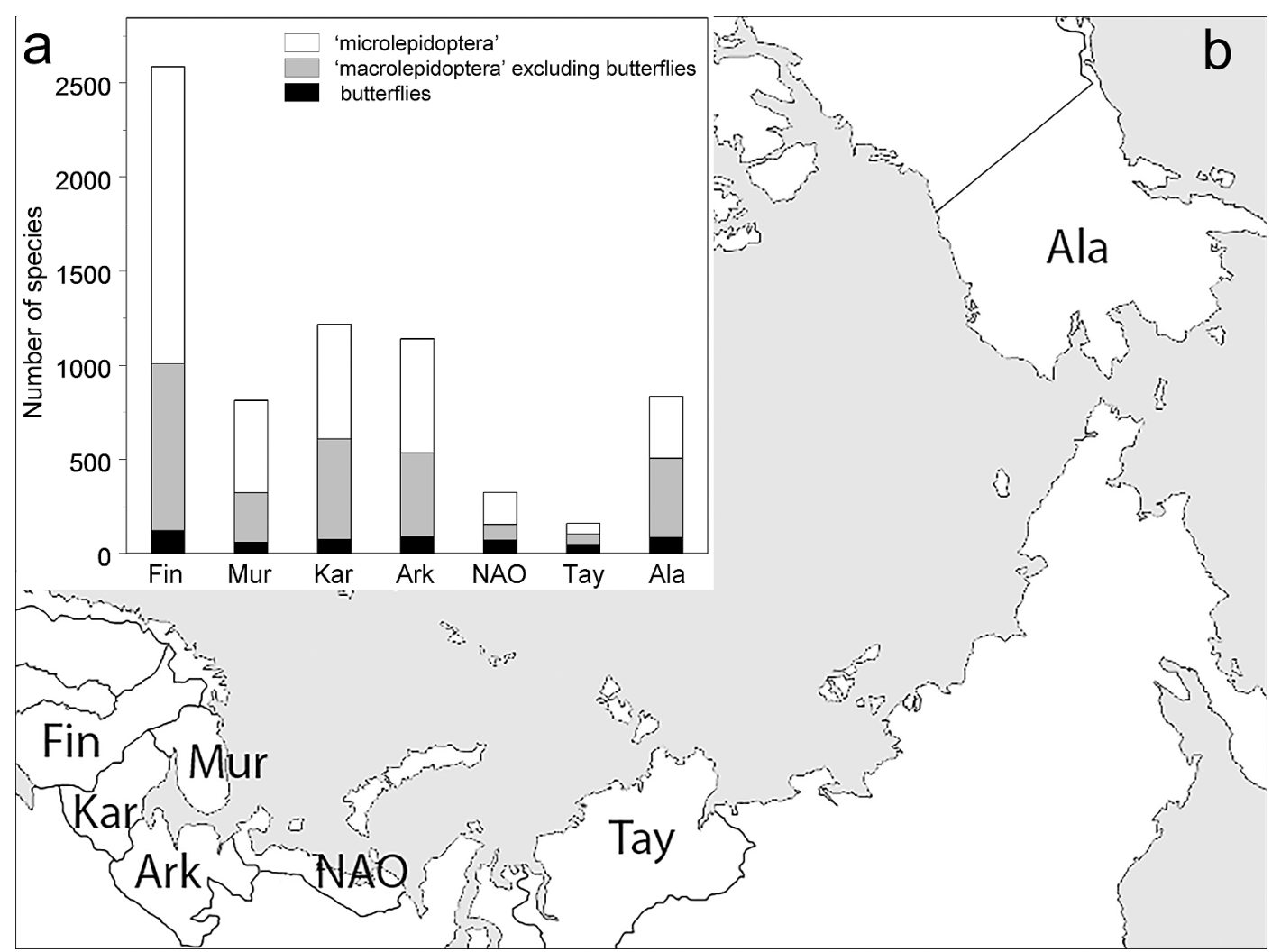

Fig. 3. Locations and compositions of lepidopteran faunas of the Arctic and Subarctic continental regions.

- a. Numbers of Lepidoptera species with subdivision to "microleipidoptera", "macroleipidoptera" and butterflies.

- b. Nenets Autonmous Okrug relative to other regions, with which the lepidopteran fauna of the former is compared. Abbreviations of the regions (source of data of Fig. 3a in parentheses): Fin, Finland (Aarvik et al. 2017); Kar, Republic of Karelia, Russia (Sinev 2008); Mur, Murmansk oblast, Russia (Kozlov \& Kullberg 2010); Ark, Arkhangelsk oblast, Russia (Kozlov et al. 2014, 2017); NAO, continental part of the Nenets Autonomous Okrug, Russia (present study); Tay, Taymyr Peninsula, Russia (Kozlov et al. 2006); Ala, Alaska, USA (Pohl et al. 2018).

\subsection{Similarities between Arctic regional faunas}

Due to insufficient levels of faunistic knowledge on "microlepidoptera", we have restricted our analysis of faunistic similarity to butterflies. In terms of its composition of butterfly fauna, NAO has an intermediate position between Fennoscandia and the Taymyr peninsula, and is equally similar to both these regions (Sørenson index 0.590.65). In comparison to these regions, it differs primarily in the proportion of the Beringian species (e.g. Parnassius corybas, Boloria alaskensis, Erebia discoidalis, Oeneis melissa). When compared to the fauna of the more southern Arkhangelsk oblast (Sørenson index 0.62), the fauna of NAO differs in the presence of tundra species (e.g. Boloria chariclea, B. polaris, B. improba, Erebia pandrose).

\section{Discussion}

\subsection{The level of faunistic knowledge}

More or less complete lists of moths and butterflies are available only for a few Arctic and subArctic regions (Fig. 3b). Restricting our comparison to continental faunas we conclude that the level of faunistic knowledge decreases substantially from Fennoscandia to the East (Fig. 3a). Of course, the absolute numbers of species recorded 
in different regions depend on both their area and climate, but the ratio between "microlepidoptera" and "macrolepidoptera", which serves an index of the completeness of the faunistic inventory (Sinev 2008), decreases from 1.5-1.6 in Finland and Murmansk oblast to 1.1 in the Arkhangelsk oblast and NAO and then to $0.5-0.6$ in the Taymyr Peninsula and Alaska. The percentage of butterfly species in these faunas seems to increase towards the north, from 5 to $10 \%$; however, the question remains whether this increase reflects real changes in the structure of regional faunas or is an artefact of the level of faunistic knowledge.

We used two approaches to evaluate the completeness of the faunistic list we compiled. First, we assumed that the list of butterflies of NAO, with 70 reported species, is nearly complete. Only 42 of these species were found in NaryanMar; the remaining 28 species are either Arctic tundra residents or are limited to more southern parts of NAO. Assuming that this proportion between different faunistic elements is valid for other groups of Lepidoptera, and that the fauna of moths in the surroundings of Naryan-Mar is relatively well explored, we estimate that the fauna of NAO is likely to include 360 species of Lepidoptera. Second, we assumed that the list of so-called "macrolepidoptera" is reasonably complete. The number of the so-called "microlepidoptera" species in well-studied northern faunas exceeds the number of "macrolepidoptera" species by a factor of 1.6 (Sinev 2008). This means that the fauna of NAO is likely to include approximately 250 species of "microlepidoptera" or 400 species of moths and butterflies in total. Allowing for a 20\% underestimation of the number of "macrolepidoptera" in the current list, we accept 500 species of Lepidoptera as a realistic upper estimate of the fauna of the continental part of NAO.

\subsection{Composition of regional fauna}

The fauna of moths and butterflies of NAO clearly differs from the fauna of Fennoscandia, due to a relatively high proportion of Beringian species in NAO. It also differs from the faunas of Taymyr and Alaska by the presence of West Palaearctic species in NAO. In particular, 33 species found in NAO (i.e. $10 \%$ of the fauna) have never been recorded from Finland. Conversely, only a handful of species (for example, Boloria napaea) are relatively common in Fennoscandia but have not yet been found in NAO or to the east of NAO.

Our findings demonstrated that several Beringian species occur in regions farther west than was previously thought. In particular, the records of Greya variabilis and Clepsis moeschleriana in Naryan-Mar extended the distribution range of these species some $400 \mathrm{~km}$ to the west. Similarly, records of Nemophora ochsenheimerella, Hepialus humuli, Dichrorampha montanana and Ancylis habeleri near Naryan-Mar substantially expanded the distribution limits of these species to the north. However, it remains unclear whether these species with generally more southern distribution have occurred in NAO for millennia or whether they have recently migrated there following climate warming.

We estimate that some $80 \%$ of the Lepidoptera species recorded from NAO belong to residents of northern boreal forests and bogs. In other words, the composition of the local fauna of moths and butterflies is much more similar to the Arkhangelsk oblast of Russia (Fig. 3a), which is almost completely covered by taiga forests, than would have been expected prior our sampling. This conclusion is similar to the one made based on the investigation of aphids: Stekolstchikov (2017) concluded that the number of truly Arctic aphids in NAO is surprisingly low. However, the extent to which these conclusions are due to a much lower sampling effort in tundra relative to forests remains unclear. To date, Amderma ( 1 in Fig. 1) remains the only locality in the tundras of NAO where "microlepidoptera" have been specifically collected (Kullberg et al. 2013).

Acknowledgements. Sampling in Naryan-Mar was made possible due to financial support from Otto Malm Foundation and Percy Sladen Memorial Fund. We are grateful to A. Stekolstchikov, T. Dyaditsyna and O. Makarova for donating their samples to MZH, to S. Sinev, A. Matov, V. Mironov, A. Tatarinov and A. Lvovsky for providing information on the occurrence of some species in NAO, to E. J. van Nieukerken for identifilation of leaf miners and for barcoding of Eriocrania species, to B. Yu. Filippov for providing access to some samples collected in NAO, and to V. V. Gorbach, E. J. van Nieukerken and S. Yu. Sinev for inspiring comments to an earlier version of the manuscript. 


\section{References}

Aarvik, L. E. 2013: Fauna Europaea: Tortricidae. — In: Karsholt, O. \& van Nieukerken, E. J. (eds.), Fauna Europaea: Lepidoptera, Moths. Fauna Europaea. Version 2017.06 [www document]. URL https://fauna-eu.org (Site visited on 16 September, 2018).

Aarvik, L., Bengtsson, B. Å., Elven, H., Ivinskis, P., Jürivete, U., Karsholt, O., Mutanen, M. \& Savenkov, N. 2017: Nordic-Baltic checklist of Lepidoptera. - Norwegian Journal of Entomology. Supplement 3: 1-236.

Alipanah, H. 2015: Contribution to the knowledge of Iranian Bactrini, Olethreutini and Enarmoniini (Lepidoptera: Tortricidae, Olethreutinae). - SHILAP Revista Lepidopterologica 43: 157-168.

Blanchemain, J. 2015: Ancylis habeleri Huemer \& Tarmann, 1997 espèce nouvelle pour la France (Lep. Tortricidae Olethreutinae). - Oreina 30: 5-6.

Bolotov, I. N. 2012: The fauna and ecology of butterflies (Lepidoptera, Rhopalocera) of the Kanin Peninsula and Kolguev Island. - Entomological Review 92: 296-304. doi: https://doi.org/10.1134/S00138738120 30062

Bolotov, I. N., Gofarov, M. Y., Rykov, A. M., Frolov, A. A. \& Kogut, Y. E. 2012: Northern boundary of the range of the clouded apollo butterfly Parnassius mnemosyne (Linnaeus) (Papilionidae): climate influence or degradation of larval host plants? - Nota Lepidopterologica 36: 19-33.

Bolotov, I. N., Tatarinov, A. G., Filippov, B. Y., Gofarov, M. Y., Kondakov, A. V., Kulakova, O. I., Potapov, G. S., Zubryi, N. A. \& Spitsyn, V. M. 2015: The distribution and biology of Pararctia subnebulosa (Dyar, 1899) (Lepidoptera: Erebidae: Arctiinae), the largest tiger moth species in the High Arctic. — Polar Biology 38: 905-911. doi: https://doi.org/10.1007/s00300014-1643-2

Coulson, S. J. 2007: The terrestrial and freshwater invertebrate fauna of the High Arctic archipelago of Svalbard. - Zootaxa 1448: 41-58. doi: http://dx.doi.org/ 10.11646/zootaxa.1448.1.2

Doney, S. C., Ruckelshaus, M., Duffy, J. E., Barry, J. P., Chan, F., English, C. A., Galindo, H. M., Grebmeier, J. M., Hollowed, A. B., Knowlton, N., Polovina, J., Rabalais, N. N., Sydeman, W. J. \& Talley, L. D. 2012: Climate change impacts on marine ecosystems. Annual Review of Marine Science 4: 11-37. doi: https://doi.org/10.1146/annurev-marine-041911111611

Early, R., Bradley, B. A., Dukes, J. S., Lawler, J. J., Olden, J. D., Blumenthal, D. M., Gonzalez, P., Grosholz, E. D., Ibañez, I., Miller, L. P., Sorte, C. J. B. \& Tatem, A. J. 2016: Global threats from invasive alien species in the twenty-first century and national response capacities. - Nature Communications 7: 12485. doi: https://doi.org/10.1038/ncomms12485

Hacker, H., Ronkay, L. \& Hreblay, M. 2002: Noctuidae Europaeae, Volume 4: Hadeninae I._- Entomological Press, Sorø. 419 pp.
Hanski, I., Clobert, J. \& Reid, W. 1995: Ecology of extinctions. - In: Heywood, V. H. \& Watson, R. T. (eds.), Global Biodiversity Assessment: 232-245 . Cambridge Univ. Press, Cambridge. $\mathrm{x}+1140 \mathrm{pp}$.

Hill, J. K., Thomas, C. D., Fox, R., Telfer, M. G., Willis, S. G., Asher, J. \& Huntley, B. 2002: Responses of butterflies to twentieth century climate warming: Implications for future ranges. - Proceedings of the Royal Society B 269: 2163-2171. doi: https://doi.org/ 10.1098/rspb.2002.2134

Hultén, E. \& Fries, M. 1986: Atlas of North European vascular plants: North of the Tropic of Cancer, vol. 1-3. - Koeltz Scientific Books, Königstein, Germany. $1172 \mathrm{pp}$.

Kozlov, M. V. 1997: Family Adelidae. - In: Kononenko, V. S. (ed.), Key to the Insects of Russian Far East. Vol. V. Trichoptera and Lepidoptera, pt. 1: 374-289. Dalnauka, Vladivostok. 537 pp. [In Russian.]

Kozlov, M. V. \& Kullberg, J. 2010 [2011]: New and interesting records of Lepidoptera from the Kola Peninsula, Northwestern Russia, in 2000-2009. — Entomologica Fennica 21: 254-272.

Kozlov, M. V., Kullberg, J. \& Dubatolov, V. V. 2006: Lepidoptera of the Taymyr Peninsula, northwestern Siberia. - Entomologica Fennica 17: 136-152.

Kozlov, M. V., Kullberg, J. \& Zverev, V. 2014: Moths and butterflies (Lepidoptera) of the Arkhangelsk oblast of Russia: an annotated checklist. — Entomologica Fennica 25: 113-141.

Kozlov, M. V., Kullberg, J. \& Zverev, V. 2017: New records of Lepidoptera from Arkhangelsk oblast of Russia. - Entomologica Fennica 28: 169-182.

Kullberg, J., Filippov, B. Yu., Spitsyn, V.M., Zubrij, N. A. \& Kozlov, M. V. 2019: Moths and butterflies (Insecta: Lepidoptera) of the Russian Arctic islands in the Barents Sea.-Polar Biology (in press). doi: https:// doi.org/10.1007/s00300-018-2425-z

Kullberg, J., Filippov, B. Yu., Zubrij, N. A. \& Kozlov, M. V. 2013: Faunistic notes on Lepidoptera collected from arctic tundra in European Russia. - Nota Lepidopterologica 36: 127-136.

Kuznetsov, V. I. 1978: Tortricidae. - In: Medvedev, G. S. (ed.), Keys to the insects of the European Part of the USSR. Vol. 4, Lepidoptera. Pt. 1: 193-680. Nauka, Leningrad, 712 pp. [In Russian.]

Lewis, J. \& Sohn, J.-C. 2015: Lepidoptera: Yponomeutoidea I (Argyresthiidae, Attevidae, Praydidae, Scythropiidae, and Yponomeutidae) . (World Catalogue of Insects 12). - Brill, Leiden \& Boston. xxiv + 253 pp.

Makarova, O. L., Sviridov, A. V. \& Klepikov, M. A. 2013: Lepidoptera (Insecta) of polar deserts. - Entomological Review 93: 225-239. doi: https://doi.org/10. 1134/S0013873813020115

Morgun, D. V. 2017: New data on the distribution of butterflies (Lepidoptera: Hesperioidea et Papilionoidea) of the Kara river basin arctic tundra (Polar Urals). Russian Entomological Journal 26: 343-347. doi: https://doi.org/10.15298/rusentj.26.4.08

Parmesan, C., Ryrholm, N., Stefanescu, C., Hill, J. K., Thomas, C. D., Descimon, H., Huntley, B., Kaila, L., 
Kullberg, J., Tammaru, T., Tennent, W. J., Thomas, J. A. \& Warren, M. 1999: Poleward shifts in geographical ranges of butterfly species associated with regional warming. - Nature 399: 579-583. doi: https://doi. org/10.1038/21181

Pohl, G. R., Landry, J.-F., Schmidt, B. C., Lafontaine, J. D., Troubridge, J. T., Macaulay, A. D., van Nieukerken, E. J., de Waard, J. R., Dombroskie, J. J., Klymko, J., Nazari, V., Stead, K. 2018: Annotated checklist of the moths and butterflies (Lepidoptera) of Canada and Alaska. - Pensoft, Bulgaria. 580 pp.

Poppius, B. R. 1906: Beiträge zur Kenntnis der Lepidopteren-Fauna der Halbinsel Kanin. - Acta Societatis pro Fauna et Flora Fennica 28: 1-11.

Ross, M. 2000: Butterflies, bumblebees and wasps. - In: van Eerden, M. R. (ed.), Pechora delta: Structure and dynamics of Pechora delta ecosystems (1995-1999): 139-145. Institute for Inland Water Management and Waste Water Treatment (RIZA), Lelystad, the Netherlands. 367 pp.

Schmid, J. 2016: Argyresthia svenssoni Bengtsson \& Johansson, 2012; Erstfunde für die Schweiz (Lepidoptera: Argyresthiidae). — Entomo Helvetica 9: 147148.

Sinev, S. Yu. (ed.) 2008: Catalogue of the Lepidoptera of Russia. - KMK Scientific Press, St. Petersburg \& Moscow. 424 pp. [In Russian.]

Stekolstchikov, A. V. 2017: Contribution to the aphid fauna (Hemiptera: Sternorrhyncha, Aphidomorpha) of the Nenets Autonomous Okrug. - Entomologist's Monthly Magazine 153: 131-155.

Sørensen, T. 1948: A method of establishing groups of equal amplitude in plant sociology based on similarity of species and its application to analyses of the vegetation on Danish commons. - Kongelige Danske Videnskabernes Selskab 5(4): 1-34.

Tatarinov, A. G. 2006a: Parnassius phoebus (Fabricius, 1793). — In: Matveeva, N. V. (ed.), Red Book of Nenets Autonomous Okrug: 266-267. Nenets Information and Analytical Centre, Naryan-Mar. 448 pp. [In Russian.]

Tatarinov, A. G. 2006b: Colias tyche (Böber, 1812). - In: Matveeva, N. V. (ed.), Red Book of Nenets Autonomous Okrug: 266-269. Nenets Information and Analytical Centre, Naryan-Mar. 448 pp. [In Russian.]

Tatarinov, A. G. 2006c: Saturnia pavonia (Linnaeus, 1758). - In: Matveeva, N. V. (ed.), Red Book of Nenets Autonomous Okrug: 266-275. Nenets Information and Analytical Centre, Naryan-Mar. 448 pp. [In Russian.]

Tatarinov, A. G. 2016: Geography of butterflies of the Eu- ropean North-East of Russia. - KMK Scientific Press, Moscow. 254 pp. [In Russian.]

Tatarinov, A. G. \& Dolgin, M. M. 1999: Diurnal Lepidopterans. (Fauna of the Northeastern Part of European Russia, vol 7, pt 1). — Nauka, St. Petersburg. 181 pp. [In Russian.]

Tatarinov, A. G. \& Gorbunov, P. Yu. 2014: Structure and spatial organization of the butterfly fauna (Lepidoptera, Rhopalocera) of the Ural Mountains. - Zoologicheskij Zhurnal 93: 108-128. [In Russian].

Tatarinov, A. G. \& Kulakova, O. I. 2005: Butterflies (Lepidoptera, Rhopalocera) of the Bolshezemelskaya Tundra in the northeast of the European part of Russia. Euroasian Entomological Journal 4: 331-337. [In Russian, English abstract.]

Tatarinov, A. G. \& Kulakova, O. I. 2010: Local faunas of butterflies (Lepidoptera: Papilionoidea, Hesperioidea) of the European North of Russia: Padimey Lakes, head reaches of the Bolshaya Rogovaya river. - Vestnik of North (Arctic) Federal University, Series Natural Sciences 0(1): 72-80. [In Russian, English abstract.]

Tatarinov, A. G. \& Kulakova, O. I. 2013: Lepidoptera and Odonata of Golodnaya Guba (Malozemelskaya tundra). - In: Nikolaeva, N. M. (ed.), Golodnaya Guba Lake: "Nizhnepechorskij" protected area: 22-27. Nenets Local History Museum, Naryan-Mar. 64 pp. [In Russian.]

Tatarinov, A. G., Sedykh, K. F. \& Dolgin, M. M. 2003: Higher Heterocera. (Fauna of the Northeastern Part of European Russia, vol 7, pt 2). — Nauka, St. Petersburg. 222 pp. [In Russian.]

Vlasova, A. A., Bolotov, I. N., Gofarov, M. Yu., Zubrij, N. A., Filippov, B. Yu., Frolov, A. A. \& Akimova, I. A. 2014: Butterfly local faunas (Lepidoptera: Rhopalocera) of the European North of Russia: the North of Yugorsky Peninsula (Amderma) and Vaygach Island. Vestnik of North (Arctic) Federal University, Series Natural Sciences 0(3): 48-60. [In Russian, English abstract.]

Walther, G. R., Post, E., Convey, P., Menzel, A., Parmesan, C., Beebee, T. J. C., Fromentin, J. M., Hoegh-Guldberg, O. \& Bairlein, F. 2002: Ecological responses to recent climate change. - Nature 416: 389-395. doi: https://doi.org/10.1038/416389a

Zvereva, E. L., Hunter, M. D., Zverev, V. \& Kozlov, M. V. 2016: Factors affecting population dynamics of leaf beetles in a subarctic region: the interplay between climate warming and pollution decline. - Science of the Total Environment 566-567: 1277-1288. doi: https:// doi.org/10.1016/j.scitotenv.2016.05.187 